\title{
Supernova Remnant G106.3+2.7: a likely proton PeVatron
}

\author{
Ruo-Yu Liu, ${ }^{a, *}$ Chong Ge, ${ }^{b}$ Shu Niu, ${ }^{c}$ Yang Chen ${ }^{a}$ and Xiang-Yu Wang ${ }^{a}$ \\ ${ }^{a}$ School of Astronomy and Space Science, Nanjing University, 210023 Nanjing, Jiangsu, China \\ ${ }^{b}$ Department of Physics and Astronomy, University of Alabama in Huntsville, Huntsville, AL 35899, USA \\ ${ }^{c}$ University of Chinese Academy of Sciences (CAS), Beijing 100049, China \\ E-mail: ryliu@nju.edu.cn
}

Supernova remnants (SNR) have long been considered as the major sources of Galactic cosmic rays (CRs). The standard shock acceleration theory predicts SNRs of fast shock, which are usually younger than a few hundred years old, can accelerate protons up to PeV. However, there is few evidence supporting acceleration of PeV CR protons in SNRs. Recently, we found the presence of X-ray synchrotron radiation from SNR G106.3+2.7. It implies a high-speed shock operating in this SNR despite it is likely already at the middle-aged stage of about $10 \mathrm{kyr}$. Combining the previous study on the atomic cloud and molecular cloud distribution in this region and the multiwavelength spectral energy distribution with our X-ray analysis, we suggest that SNR G106.3+2.7 is likely a proton PeVatron.

$37^{\text {th }}$ International Cosmic Ray Conference (ICRC 2021)

July 12 th - 23rd, 2021

Online - Berlin, Germany

\footnotetext{
*Presenter
} 


\section{Introduction}

The local CR spectrum roughly shows a power-law behaviour up to the so-called knee around $1 \mathrm{PeV}$. It implies the existence of powerful proton petaelectronvolt accelerators (PeVatrons) residing in our Galaxy. SNRs have long been suggested as the main sources of Galactic CRs. According to the diffusive shock acceleration theory [1,2], which is widely accepted as the promising mechanism for $\mathrm{CR}$ acceleration, the acceleration rate of particles highly depends on the shock velocity. Therefore, young SNRs within a few hundred years are considered as the sources of $\mathrm{PeV}$ protons[3], since their shocks have not been severely decelerated yet by the swept-up interstellar medium. An important indicator of the fast shock is the synchrotron X-ray radiation. Indeed, many young SNRs such as SNR RX J1713.7-3946[4], SNR G1.9+0.3[5], Tycho[6], Cassiopeia A[7], Kepler[8] and etc show synchrotron X-ray radiation. However, in multi-TeV gamma-ray band, people have not found direct and decisive evidence supporting these SNRs as proton PeVatron.

SNR G106.3+2.7 is associated with the Boomerang Nebula. The system shows a highly asymmetric radio morphology with a compact, bright head in northeast and an elongated, faint tail extending towards southwest[9]. The Boomerang Nebula, located in the northeast part of the system, is powered by an energetic pulsar PSR J2229+6114 with a present rotation period of $51.6 \mathrm{~ms}$ and a spindown luminosity $2.2 \times 10^{37} \mathrm{erg} / \mathrm{s}[10]$. The characteristic age of the pulsar is about $10 \mathrm{kyr}$ which is also considered as a representation of the SNR's age[11], although the true age can be shorter than the characteristic age if the initial rotation period is close to the present rotation period. The system resides in an intriguing environment where the head seems to be enveloped by dense HI clouds while the tail is expanding into a low-density cavity which is probably excavated by the stellar wind of previous generations of massive stars[12]. In addition to the HI clouds, a dense molecular cloud also appears in the tail region of the SNR at approximately the same distance[12]. An analysis with CO-line data of IRAM $30 \mathrm{~m}$ telescope towards the tail region does not find clear evidence of the direct interaction between the SNR and the molecular cloud ${ }^{1}$, so that the molecular cloud is probably close to but not contiguous to the SNR. Interestingly, gamma-ray emission from $\mathrm{GeV}$ to multi-hundred $\mathrm{TeV}$ is detected in the SNR tail region and is in spatial coincidence with the molecular cloud [13-17], which implies a hadronic origin of the gamma-ray emission and subsequently the presence of PeVatron in this system. Previous studies have shown that either an a leptonic interpretation or a hadronic interpretation can explain the SED, but the predicted X-ray flux is quite different.

Recently, we did a detailed analysis on the X-ray data of Chandra, XMM-Newton and Suzaku in this region [18]. If jumping to the conclusion, we found that, surprisingly, X-ray synchrotron emission occurs in the tail region of this middle-aged SNR, which is most likely emitted by fresh electrons accelerated by the SNR shock in the tail region, according to the X-ray surface brightness profile. We then inferred that the particle acceleration rate of the SNR shock in the tail is capable of energizing protons to $\mathrm{PeV}$. On the other hand, the X-ray flux put a limit to the multi-TeV flux contributed by the inverse Compton (IC) scatterings of electrons and a hadronic component is needed to explain the multi-TeV flux up to $100 \mathrm{TeV}$, corroborating that SNR G106.3+2.7 is a proton PeVtron. The rest of this paper is organized as follows: in Section 2 we introduce the X-ray data

${ }^{1}$ Q. C. Liu \& Y. Chen, to be submitted 
analysis and the results; we interpret the result and discuss the physical implication in in Section 3; we make a conclusion with a brief outlook in Section 4. For details, we refer readers to Ref.[18]

\section{X-ray data analysis}

To obtain the spectral information in different parts of the SNR (marked as HC, OC, HX, TX, OX1, OX2, HS, TS1 and TS2, where "H" stands for head, "T" for tail, "O" for outside, "C" for Chandra, "X" for XMM-Newton, and "S" for Suzaku), we extracted spectra from Chandra, $X M M$-Newton, and Suzaku in regions (with X-ray point sources masked) as shown in Fig. 1. We also extract a diffuse sky background spectrum from the ROSAT All-Sky Survey (RASS) in a 1-2 degree annulus surrounding the SNR. We jointly fitted the spectral data of RASS with that from Chandra, XMM-Newton, and Suzaku respectively to link the sky background in different analyses, which is modelled by two thermal components for the foreground Galactic soft X-ray emission and one power-law component for the unresolved cosmic X-ray background. Distinct contrasts of surface brightness inside and outside the SNR can be seen, as shown in Table 1. The best-fit spectra from these regions inside the SNR are consistent with a featureless power-law (PL), as is later confirmed by an independent analysis with Suzaku[19], while the X-ray emission outside the SNR is insignificant and consistent to the sky background fluctuation. The best-fit hydrogen column density is $N_{\mathrm{H}} \sim 0.95 \times 10^{22} \mathrm{~cm}^{-2}$.

To investigate the origin of the X-ray emission in different parts of the SNR, we extracted a radial intensity profile and a spectral profile in a series of annulus sectors, respectively, as shown in Fig. 2. Our analysis towards the Boomerang region confirms the results of the previous study. The emission of the nebula is largely confined within a radius of $100^{\prime \prime}$ with a rapid intensity decline towards large radius. Fainter nonthermal X-ray emission permeates throughout the entire head region outside the PWN. The intensity in this region also decreases with the distance from the pulsar but in a shallower manner, which is accompanied by a gradual softening of the X-ray spectrum. Such a spatial tendency implies that the X-ray emission in the head of the SNR is related to the PWN, probably due to electrons escaping the PWN. In the tail region, interestingly, both the intensity profile and the spectral profile becomes flat. Such a change suggests a different origin of the X-ray emission in the tail region from those in the head region. Considering the rapid cooling of the X-ray-emitting electrons, these electrons are most likely accelerated directly in the tail region. The most plausible accelerator in the tail region is the SNR shock.

\section{Interpretation}

According the the diffusive shock acceleration theory [1,2], the acceleration timescale highly depends on the shock speed, which reads[20,21]

$$
t_{\mathrm{acc}} \approx 4 \eta^{-1}\left(\frac{E}{1 \mathrm{TeV}}\right)\left(\frac{B}{10 \mu \mathrm{G}}\right)^{-1}\left(\frac{v_{s}}{3000 \mathrm{~km} \mathrm{~s}^{-1}}\right)^{-2} \mathrm{yr}
$$

where $E$ is the particle energy, $B$ is the magnetic field in the downstream region and $\eta(\leq 1)$ represents the acceleration efficiency. For electrons, the acceleration is generally limited by the cooling of synchrotron radiation. Equating the acceleration timescale to the synchrotron cooling 


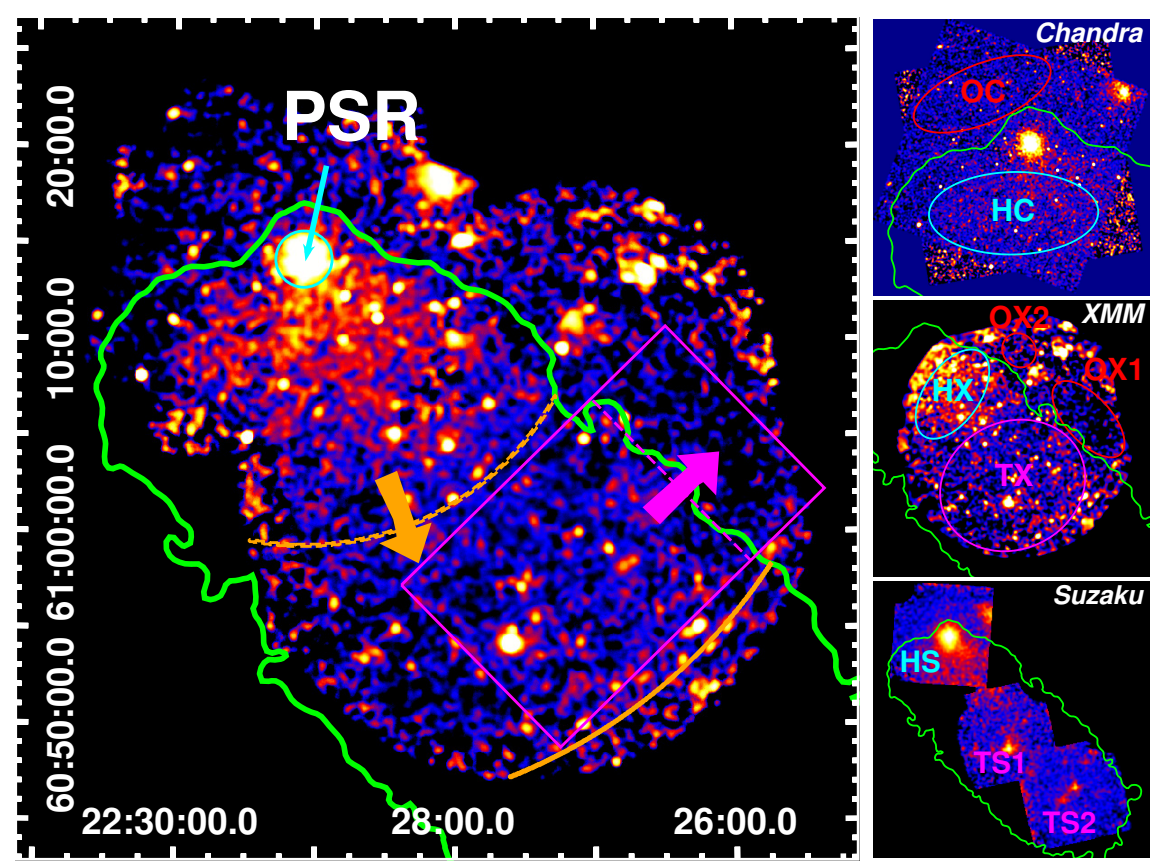

Figure 1: Left: Combined Chandra and XMM-Newton instrumental background subtracted, exposure corrected image in $1-7 \mathrm{keV}$. The green curve outlines the SNR observed by $1.4 \mathrm{GHz}$ radio continuum[9]. The cyan circle has a radius of 100 " centred at the position of PSR J2229+6114, characterising the boundary of the Boomerang Nebula. The dashed orange arc marks the approximate boundary between the head and the tail of the SNR in X-ray band, based on the SBP extracted from a series of annuli sectors centred at PSR J2229+6114 out to the position marked with the solid orange arc (see Fig. 2A). The magenta box is the intensity extraction region to show the brightness change at the boundary of the SNR along the direction of arrows (see Fig. 2B). Right: Chandra, XMM-Newton, and Suzaku images in 1-7 keV with marked regions for spectral analysis in Table 1. $\mathrm{H}$ is short for head, $\mathrm{T}$ is for tail, and $\mathrm{O}$ is for outside the SNR; $\mathrm{C}$ is for Chandra, $\mathrm{X}$ is for XMM-Newton, and $\mathrm{S}$ is for Suzaku. The green contours outlines the SNR.

timescale, one can obtain the maximum achievable electron energy in the cooling-limited scenario and subsequently arrive at that the synchrotron spectral cutoff energy of the electrons accelerated by the shock is only dependent on the shock speed[e.g., 22, 23], i.e.,

$$
\epsilon_{\mathrm{syn}, \max } \approx 7 \eta\left(v_{s} / 3000 \mathrm{~km} \mathrm{~s}^{-1}\right)^{2} \mathrm{keV}
$$

The continuation of the X-ray spectrum up to $7 \mathrm{keV}$ without a clear spectral cutoff suggests a high speed for the SNR shock in the tail region which is $v_{s} \geq 3000 \eta^{-1 / 2} \mathrm{~km} / \mathrm{s}$.

Generally speaking, for a middle-aged SNR of about $10 \mathrm{kyr}$, this shock speed would be unreasonably high. However, considering that the tail region is formed by the SNR shock breaking out into a low-density cavity, it is possible that the shock is not significantly decelerated and maintains a high speed. What's more, the inferred velocity is also consistent with the elongated morphology of the SNR tail. The projected length of the tail is $L_{\mathrm{t}} \simeq 14(d / 800 \mathrm{pc}) \mathrm{pc}$ assuming the supernova explosion center is close to the position of PSR J2229+6114[12]. Therefore, the mean projected shock speed in the tail region can be inferred independently as $v_{s} \sim L_{\mathrm{t}} / T_{\text {age }} \simeq$ $1500 \mathrm{~km} \mathrm{~s}^{-1}(d / 800 \mathrm{pc})\left(T_{\text {age }} / 10 \mathrm{kyr}\right)^{-1}$. Note that the true shock velocity can be several times higher since the velocity inferred in this way is only the component projected on the plane of the 

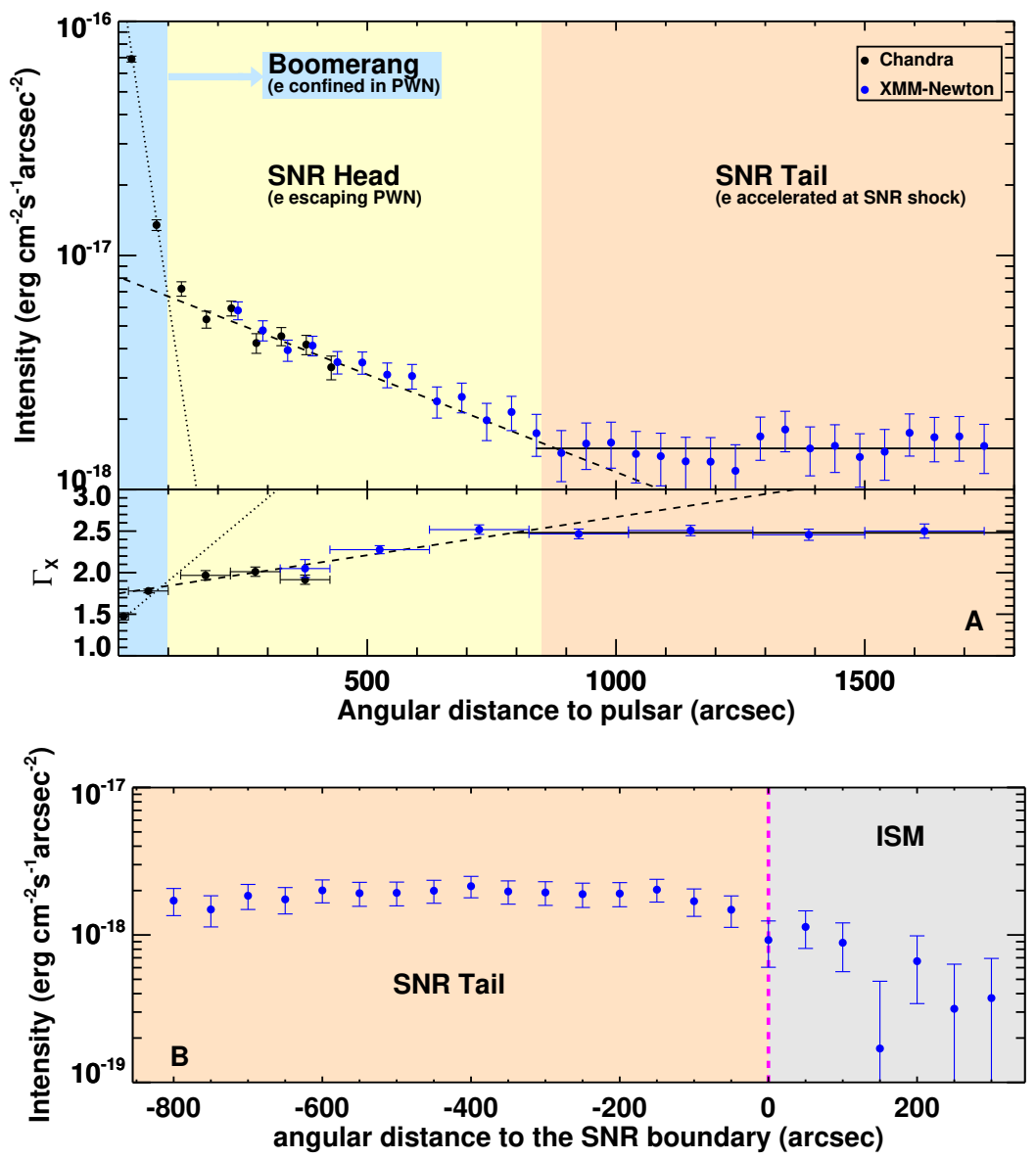

Figure 2: Upper: Radial profiles of X-ray surface brightness and spectral index extracted from a series of annuli centred at PSR J2229+6114. The central bright point source, i.e. the pulsar's emission, has been subtracted. The dotted, dashed, solid lines represent a PWN component, an escaping electron component in the head region and an SNR component in the tail region, respectively. Lower: One-dimensional X-ray surface brightness profile inside the magenta rectangle shown in Fig. 1, after averaging over the short edge of the rectangle. The $\mathrm{x}$-axis follows the direction of the magenta arrow. The dashed magenta line marks the boundary that separates the SNR tail and the interstellar medium (ISM), corresponding to position marked also with the dashed magenta line in Fig. 1.

sky. Additionally, a larger mean velocity can be obtained in this way, if the age of the system turns out to be shorter than the characteristic age of the pulsar.

Because the energy loss rate of protons is much slower than that of equal-energy electrons, the proton acceleration is age-limited [24]. Substituting Eq. (2) into Eq. (1), the maximum proton energy achievable in the SNR shock of a dynamical age of $T_{\text {age }}$ can be given by

$$
E_{p, \max } \approx 3\left(\frac{T_{\text {age }}}{10 \mathrm{kyr}}\right)\left(\frac{B}{10 \mu \mathrm{G}}\right)\left(\frac{\epsilon_{\mathrm{syn}, \mathrm{max}}}{7 \mathrm{keV}}\right) \mathrm{PeV} .
$$

It implies that the SNR G106.3+2.7 can serve as proton PeVatron as long as the magnetic field is not too weak.

On the other hand, to interpret the SED of the tail region, a hadronic component is also needed with the proton spectrum extending up to $\sim 1 \mathrm{PeV}$. Fitting the radio and X-ray spectrum 


\begin{tabular}{lccc}
\hline \hline Reg $^{a}$ & PL index & Intensity $^{b}\left(\mathrm{erg} \mathrm{cm}^{-2} \mathrm{~s}^{-1} \mathrm{arcmin}^{-2}\right)$ & $\chi^{2} / \mathrm{DOF}$ \\
\hline PWN & $1.7 \pm 0.1$ & $(1.1 \pm 0.1) \times 10^{-13}$ & $307.6 / 303$ \\
\hline $\mathrm{HC}$ & $1.9 \pm 0.1$ & $(1.3 \pm 0.1) \times 10^{-14}$ & $734.9 / 606$ \\
$\mathrm{HX}$ & $2.2 \pm 0.1$ & $(1.3 \pm 0.1) \times 10^{-14}$ & $230.7 / 192$ \\
$\mathrm{HS}$ & $2.0 \pm 0.1$ & $(1.2 \pm 0.1) \times 10^{-14}$ & $207.6 / 240$ \\
\hline $\mathrm{TX}$ & $2.4 \pm 0.1$ & $(5.4 \pm 0.5) \times 10^{-15}$ & $1122.0 / 892$ \\
$\mathrm{TS} 1$ & $2.0 \pm 0.1$ & $(7.2 \pm 0.4) \times 10^{-15}$ & $98.8 / 113$ \\
$\mathrm{TS} 2$ & $2.0 \pm 0.1$ & $(5.7 \pm 0.3) \times 10^{-15}$ & $181.5 / 166$ \\
\hline OC & $2.6 \pm 0.5$ & $(1.3 \pm 0.9) \times 10^{-15}$ & $399.6 / 364$ \\
OX1 & $4.5 \pm 0.7$ & $(1.0 \pm 0.8) \times 10^{-15}$ & $224.6 / 185$ \\
OX2 & $5.8 \pm 1.2$ & $(1.9 \pm 1.8) \times 10^{-15}$ & $156.9 / 134$ \\
\hline BKG & - & $(5.6 \pm 1.0) \times 10^{-15}$ & - \\
\hline & & &
\end{tabular}

Table 1: Spectral Properties in Different Regions of SNR G106.3+2.7.

${ }^{a}$ PWN represents the emission within $100^{\prime \prime}$ of the pulsar but excluding the emission of the pulsar itself. H stands for the head, T for the tail, and O for outside SNR; C stands for Chandra, X for XMM-Newton, and S for Suzaku. The corresponding spectral extraction regions are marked in Figure 1. BKG stands for sky background intensity.

$b$ Intensity in $1-7 \mathrm{keV}$.

requires a broken power-law function for the electron spectrum. The X-ray spectrum index in the tail region is 2.4 so the spectral index of electrons responsible for the X-ray emission is 3.8. These electrons also emit gamma rays above $\mathrm{TeV}$ via the IC radiation. However, given such a soft spectrum and the Klein-Nishina effect, the resulting IC spectrum is too soft to account for the data. As a consequence, a hadronic gamma-ray component from the interactions between escaping CR protons and the molecular cloud has to be introduced to explain the spectrum of the tail region at least above $10 \mathrm{TeV}$ (or even above $\mathrm{GeV}$ if a comparatively high magnetic field is assumed, see Fig. 3), because there are no other reasonable particle accelerators in this region. The required proton spectrum is harder than the one predicted in the DSA theory, probably due to the propagation effect, where higher-energy CRs have escaped and reached the cloud while lower-energy CRs are still confined in the shock [25].

\section{Discussion and Conclusion}

SNR G106.3+2.7 is the first supernova remnant showing synchrotron X-ray emission. Even though it is probably already in the middle-age stage, the nonthermal X-ray radiation from the tail region indicates that its shock velocity still remains at several thousand kilometers per second. This is most likely due to the special environment it resides, where the southwest part of the SNR breaks into a low-density cavity, as supported by the elongated morphology. Its shock velocity in the tail region is much higher when compared with other middle-aged SNRs, so a high particle acceleration rate is expected. In addition, it has experienced a much longer lifetime when compared with young SNRs that have high shock velocity, offering sufficient time for the CRs to be gradually accelerated up to PeV energies. Our study may challenge the present SNR paradigm of CR origin, and we speculate that a special category of SNRs, which are at middle-aged stage and located in 

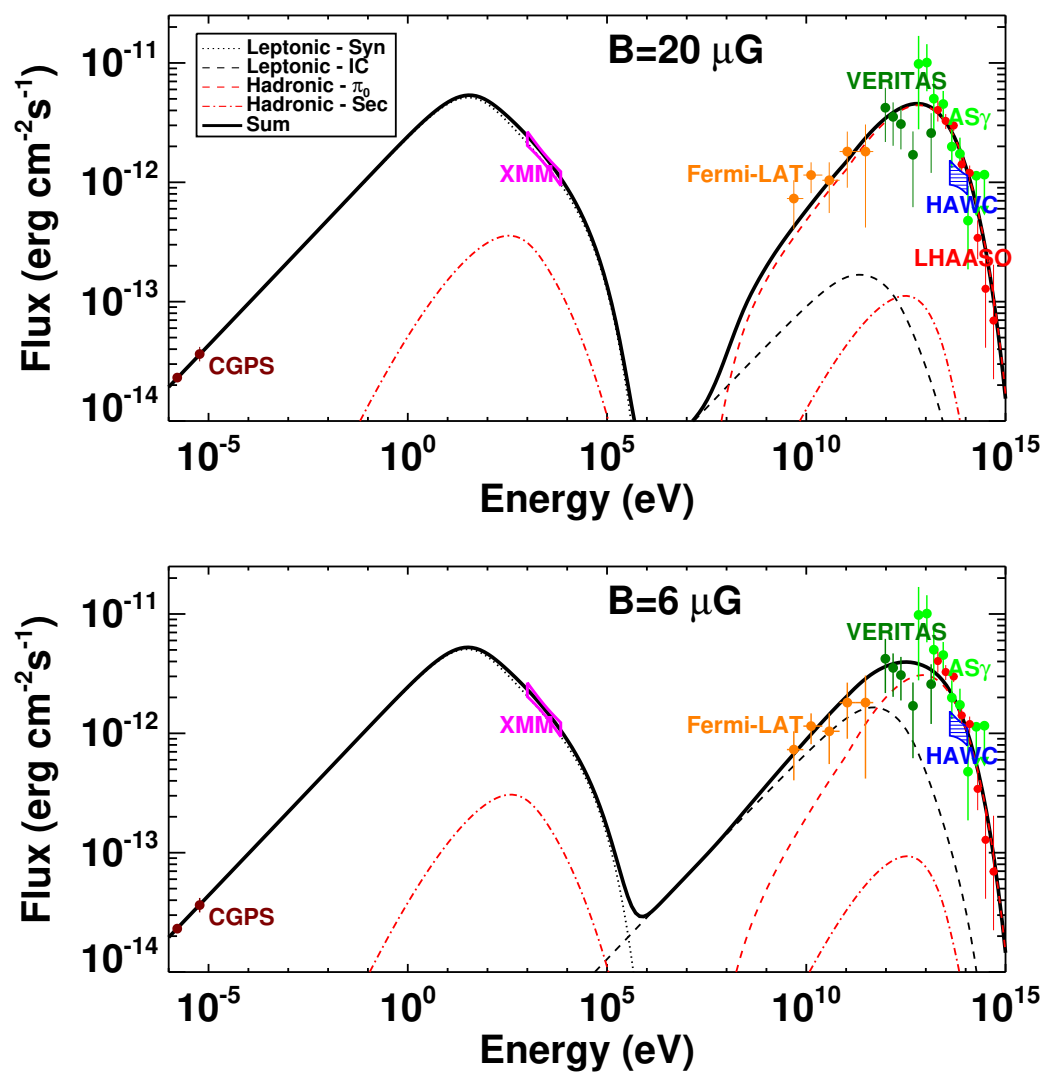

Figure 3: Modelling of multiwavelength spectrum of the tail region of SNR G106.3+2.7, assuming a magnetic field of $B=$ $20 \mu \mathrm{G}$ (upper panel) and $B=6 \mu \mathrm{G}$ (lower panel). In both panels, dotted and dashed black curves represent, respectively, the synchrotron radiation and the IC radiation of electrons. Dashed red curves show the pionic gamma-ray emission from $p p$ collisions of CR protons while dot-dashed red curves show the emission of secondary electrons/positrons co-produced in $p p$ collisions. The thick solid black curves show the sum of these components. Model parameters for the case of $B=20 \mu \mathrm{G}$ : total electron energy $W_{e}=3 \times 10^{46} \mathrm{ergs}$, electron spectral break energy $E_{e, b}=5 \mathrm{TeV}$, total proton energy $W_{p}=8 \times 10^{46} \mathrm{ergs}$, proton spectral break energy $E_{p, b}=200 \mathrm{TeV}$, proton spectral index before the break $\alpha_{p}=1.6$, the index change after the break $\Delta \alpha_{p}=1$; Model parameters for the case of $B=6 \mu \mathrm{G}: W_{e}=2.3 \times 10^{47} \mathrm{ergs}, E_{b}=9 \mathrm{TeV}$, $W_{p}=5 \times 10^{46} \mathrm{ergs}, E_{p, b}=200 \mathrm{TeV}, \alpha_{p}=1.5, \Delta \alpha_{p}=1$; for both two cases, the electron spectral index before the break $\alpha_{e}=2.3$ and the index change after the break is $\Delta \alpha_{e}=1.4$, the maximum electron energy $E_{e, \max }=0.5 \mathrm{PeV}$ and the maximum proton energy is $E_{p, \max }=1 \mathrm{PeV}$. The gas density for the hadronuclear interaction is assumed to be $50 \mathrm{~cm}^{-3}$. The CGPS data follows Ref.[9]. The Fermi-LAT data is analysed in Ref.[14]. The VERITAS data is taken from Ref.[13]. The HAWC data is taken from Ref.[15]. The Tibet AS $\gamma$ data is taken from Ref.[16] The LHAASO data is taken from Ref.[17]. The X-ray bowtie is obtained in this work by multiplying the intensity (flux per solid angle) of the TX region (see Table. 1) with a solid angle of $600 \mathrm{arcmin}^{2}$ approximately for the entire tail region.

low-density cavity, are proton PeVatrons. However, whether such a special category of SNRs can make the major contribution to the measured PeV CRs would depend on how rare they are in the Galaxy, which is to be studied in the future.

\section{References}

[1] A. R. Bell, MNRAS 182, 147 (1978).

[2] R. Blandford and D. Eichler, Phys. Rep. 154, 1 (1987). 
[3] K. M. Schure and A. R. Bell, MNRAS 435, 1174 (2013), 1307. 6575.

[4] K. Koyama, K. Kinugasa, K. Matsuzaki, M. Nishiuchi, M. Sugizaki, K. Torii, S. Yamauchi, and B. Aschenbach, PASJ 49, L7 (1997), astro-ph/9704140.

[5] S. P. Reynolds, K. J. Borkowski, D. A. Green, U. Hwang, I. Harrus, and R. Petre, ApJ 680, L41 (2008), 0803 . 1487.

[6] U. Hwang, A. Decourchelle, S. S. Holt, and R. Petre, ApJ 581, 1101 (2002), astro-ph/0208485.

[7] E. V. Gotthelf, B. Koralesky, L. Rudnick, T. W. Jones, U. Hwang, and R. Petre, ApJ 552, L39 (2001), astro-ph/ 0104161.

[8] S. P. Reynolds, K. J. Borkowski, U. Hwang, J. P. Hughes, C. Badenes, J. M. Laming, and J. M. Blondin, ApJ 668, L135 (2007), 0708 . 3858.

[9] S. Pineault and G. Joncas, AJ 120, 3218 (2000).

[10] R. N. Manchester, G. B. Hobbs, A. Teoh, and M. Hobbs, AJ 129, 1993 (2005), astro-ph/0412641.

[11] J. P. Halpern, F. Camilo, E. V. Gotthelf, D. J. Helfand , M. Kramer, A. G. Lyne, K. M. Leighly, and M. Eracleous, ApJ 552, L125 (2001), astro-ph/0104109.

[12] R. Kothes, B. Uyaniker, and S. Pineault, ApJ 560, 236 (2001), astro-ph/0106270.

[13] V. A. Acciari, E. Aliu, T. Arlen, T. Aune, M. Bautista, M. Beilicke, W. Benbow, D. Boltuch, S. M. Bradbury, J. H. Buckley, et al., ApJ 703, L6 (2009), 0911.4695.

[14] Y. Xin, H. Zeng, S. Liu, Y. Fan, and D. Wei, ApJ 885, 162 (2019), 1907. 04972.

[15] A. Albert, R. Alfaro, C. Alvarez, J. R. A. Camacho, J. C. Arteaga-Velázquez, K. P. Arunbabu, D. Avila Rojas, H. A. Ayala Solares, V. Baghmanyan, E. Belmont-Moreno, et al., ApJ 896, L29 (2020), 2005 . 13699.

[16] Tibet AS $\gamma$ Collaboration, M. Amenomori, Y. W. Bao, X. J. Bi, D. Chen, T. L. Chen, W. Y. Chen, X. Chen, Y. Chen, S. W. Cirennima, Cui, et al., Nature Astronomy (2021).

[17] Z. Cao, F. A. Aharonian, Q. An, L. X. Axikegu, Bai, Y. X. Bai, Y. W. Bao, D. Bastieri, X. J. Bi, Y. J. Bi, H. Cai, et al., Nature 594, 33 (2021).

[18] C. Ge, R.-Y. Liu, S. Niu, Y. Chen, and X.-Y. Wang, The Innovation 2, 100118 (2021), 2012.11531.

[19] Y. Fujita, A. Bamba, K. K. Nobukawa, and H. Matsumoto, ApJ 912, 133 (2021), 2101. 10329.

[20] J. G. Kirk and R. O. Dendy, Journal of Physics G Nuclear Physics 27, 1589 (2001), astro-ph/0101175.

[21] F. M. Rieger, V. Bosch-Ramon, and P. Duffy, Ap\&SS 309, 119 (2007), astro-ph/0610141.

[22] E. Parizot, A. Marcowith, J. Ballet, and Y. A. Gallant, A\&A 453, 387 (2006), astro-ph/0603723.

[23] V. N. Zirakashvili and F. Aharonian, A\&A 465, 695 (2007), astro-ph/0612717.

[24] A. R. Bell, K. M. Schure, B. Reville, and G. Giacinti, MNRAS 431, 415 (2013), 1301. 7264.

[25] Y. Bao and Y. Chen, arXiv e-prints arXiv:2103.01814 (2021), 2103.01814. 